\title{
Career Development
}

\section{Brad Conrad \\ Society of Physics Students}

This session will feature resume/CV critiques and mock interviews (by preschedule only on the Meeting Registration Form) for early career scientists. Effective communication of one's skills and abilities to a broad audience is a necessary skill but one that is frequently not discussed within an academic's career. To address this, this professional development session will bring together experts of varied career paths to work one-on-one with young scientists (who have preregistered for the event) to examine how they can improve their written and verbal communication skills to potential employers. After this, a speaker will facilitate an interactive session for an open audience about presenting one's studies and research effectively in a resume/CV and interview. 